\title{
Scalar and density concomitants of tensor with the valence $(1,2)$ in a 2-dimensional space
}

\author{
by S. WEcGRZYNOWsKI (Szczecin)
}

1. Introduction. Suppose we are given an abstract special purely differential geometric object [9] with a fibre $\mathfrak{M}$ and with the transformation formula :

$$
\omega^{\prime}=\mathbb{F}(\omega, L) ; \quad \omega \in \mathfrak{M}, L \in \mathscr{L}_{s}^{n} .
$$

The subset $\mathfrak{M}$ of the fibre $\mathfrak{M}$ is called an allowable set $[8]$ if the implication

$$
\bigwedge_{\omega_{1} \in \overline{\mathbb{M}}} \bigwedge_{L \in \mathscr{S}_{s}^{n}}\left(\omega_{1} \in \overline{\mathfrak{M}} \Rightarrow \omega_{2}=F\left(\omega_{1}, L\right) \in \overline{\mathfrak{M}}\right)
$$

holds.

An allowable set is the union sum of transitive fibres.

Suppose we are given the geometric object (1.1). Let us take the subobject $\bar{\omega}$ of the object $\omega$ with respect to the allowable set $\overline{\mathfrak{M}} \subset \mathfrak{M}$ [10]. Every concomitant of the subobject $\bar{\omega}$ is called the relative concomitant of the object $\omega$ with respect to the allowable set $\overline{\mathfrak{M}}$ [8].

The purpose of this paper is to determine all scalar concomitants of a tensor $t_{\lambda \mu}^{\nu}$ in the 2-dimensional space. The fibre of the tensor $t_{\lambda \mu}^{\nu}$ has been divided into allowable sets in which the relative scalar concomitants have been determined. In Section 7 there are given some remarks concerning the density concomitants of the tensor $t_{\lambda \mu}^{\nu}$.

2. The allowable sets of a tensor $t_{\lambda \mu}^{\nu}$ in a two-dimensional space. Suppose we are given the tensor $t_{\lambda \mu}^{\nu}$ at some point $x_{0}$ of the two-dimensional manifold of class $C^{1}$ with the transformation formula:

$$
t_{\lambda^{\prime} \mu^{\prime}}^{\nu^{\prime}}=A_{\lambda^{\prime}}^{\lambda} A_{\mu^{\prime}}^{\mu} A_{\nu}^{\nu^{\prime}} t_{\lambda \mu}^{\nu} \quad\left(\lambda, \mu, \nu=1,2 ; \lambda^{\prime}, \mu^{\prime}, \nu^{\prime}=1^{\prime}, 2^{\prime}\right) .
$$

The whole space $R^{8}$ is the fibre $\mathfrak{M}$ of $t_{\lambda \mu}^{v}$. The group $\mathscr{L}_{1}^{2}$ acting in our problem is a group of non-singular square matrices of the second order.

In the sequel we introduce some division of the fibre $\mathfrak{M}$ of $t_{\lambda \mu}^{\nu}$ into allowable sets. In these sets we shall determine the relative scalar concomitants of $t_{\lambda \mu}^{\nu}$. 
Let us consider two covariant vectors formed by the contraction of the tensor $t_{\lambda \mu}^{\nu}$ :

$$
v_{\lambda} \stackrel{\mathrm{df}}{=} t_{\lambda \mu}^{\mu}, \quad \stackrel{2}{v_{\lambda}} \stackrel{\mathrm{df}}{=} t_{\mu \lambda}^{\mu} .
$$

Now let us introduce the following notation:

$$
\begin{aligned}
& \mathfrak{M}_{2}=\left\{t_{\lambda_{\mu}}^{\nu}: t_{\lambda_{\mu}}^{\nu} \epsilon \mathfrak{M}, \operatorname{det}\left\|v_{\lambda}\right\| \neq 0\right\}, \\
& \mathfrak{M}_{\varkappa \mathrm{l}}=\left\{t_{\lambda \mu}^{v}: t_{\lambda \mu}^{v} \in \mathfrak{M}, \stackrel{1}{v} \neq 0, \stackrel{2}{v}=\stackrel{1}{1}\right\} \text {, } \\
& \mathfrak{M}_{10}=\left\{t_{\lambda \mu}^{\nu}: t_{\lambda \mu}^{v} \in \mathfrak{M}, \stackrel{1}{v}=0, \quad v^{2} \neq 0\right\}, \\
& \mathfrak{M}_{00}=\left\{t_{\lambda \mu}^{\nu}: t_{\lambda \mu}^{\nu} \in \mathfrak{M}, \stackrel{1}{v}=\stackrel{2}{v}=0\right\} \text {. }
\end{aligned}
$$

The subsets $\mathfrak{M}_{2}, \mathfrak{M}_{x 1}, \mathfrak{M}_{10}$ and $\mathfrak{M}_{00}$ are the allowable sets of the fibre $\mathfrak{M}$ of $t_{\lambda \mu}^{\nu}$. On the basis of [4] and [6] it is known that all transitive fibres of the pair of vectors $v_{\lambda}$ and ${ }_{2}$ are $\mathfrak{M}_{2}, \mathfrak{M}_{x 1}, \mathfrak{M}_{10}$ and $\mathfrak{M}_{00}$. Hence it follows that the fibre $\mathfrak{M}$ is the sum of the allowable sets:

$$
\mathfrak{M}=\mathfrak{M}_{2} \cup \mathfrak{M}_{x 1} \cup \mathfrak{M}_{10} \cup \mathfrak{M}_{00} .
$$

3. The scalar concomitants of $t_{\lambda \mu}^{\nu}$ in $\mathfrak{M}_{2}$. In the case of the scalar concomitants of the tensor $t_{\lambda \mu}^{v}$ the functional equation for the required concomitant takes the form:

$$
f\left(t_{\lambda \mu}^{\nu}\right)=f\left(t_{\lambda_{\mu}}^{\nu} A_{\lambda^{\prime}}^{\lambda} A_{\mu^{\prime}}^{\mu} A_{\nu}^{\nu^{\prime}}\right), \quad t_{\lambda \mu}^{\nu} \in \mathfrak{M}, A_{\lambda}^{\lambda^{\prime}} \in \mathscr{L}_{1}^{2} .
$$

Solving equation (3.1) when $t_{\lambda \mu}^{\nu} \epsilon \mathfrak{M}_{2}$, we determine all relative scalar concomitants of $t_{\lambda \mu}^{\nu}$ in $\mathfrak{M}_{2}$. As $A$ in equation (3.1) we take the matrix:

$$
A \stackrel{\mathbf{d f}}{=}\left\|A_{\lambda}^{\varrho^{\prime}}\right\|=\left\|e^{\prime}\right\| \quad(\lambda, \varrho=1,2) ; \varrho=\varrho^{\prime} .
$$

Then the elements of the inverse matrix $v^{-1}$ are determined by the relations

$$
\underset{\sigma}{e-1} v_{\sigma}^{-1}=\delta_{\sigma}^{e}
$$

where $\delta_{\sigma}^{o}$ are Kronecker symbols. After the substitution (3.2) equation (3.1) takes the form

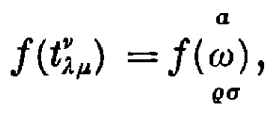

where $\omega$ are scalars

$$
\underset{\varrho \sigma}{a}=t_{\lambda \mu}^{v} v_{\varrho}^{-1-1 \alpha} v^{\mu} v_{\nu} \quad(\varrho, \sigma, \alpha=1,2) .
$$


After the transformation of the coordinate system determined by (3.2) the components of the vectors $v_{\lambda^{\prime}}$ and ${ }_{\lambda^{\prime}}^{2}$ become

$$
\stackrel{1}{v_{1^{\prime}}}=1, \quad \stackrel{1}{v_{2^{\prime}}}=0 ; \quad \stackrel{2}{v_{1^{\prime}}=0,} \quad \stackrel{2}{v_{2^{\prime}}}=1 .
$$

On account of (3.6) among eight scalars of the form (3.5) only four (i), $1, \omega$ $2 \quad \begin{array}{llll}11 & 22\end{array}$ and $\omega$ are essentially different. Thus we have proved the following 22

THEOREM 1. The most general scalar concomitant of $t_{\lambda \mu}^{y}$ in $\mathfrak{M}_{2}$ is a function of the form

$$
f\left(t_{\lambda \mu}^{\nu}\right)=\left(\begin{array}{cccc}
1 & 2 & 1 & 2 \\
\omega, & \omega, & \omega & (1)
\end{array}\right),
$$

where ${ }^{a}$ are defined in (3.5) and $\varphi$ is arbitrary.

eo

4. The scalar concomitants of $t_{\lambda \mu}^{\nu}$ in $\mathfrak{M}_{x 1}$. In $\mathfrak{M}_{x 1}$ we first of all determine some density $\mathfrak{g}$ formed from the tensor $t_{\lambda_{\mu}}^{v}$ and later we divide $\mathfrak{M}_{x 1}$ with the aid of $\mathfrak{g}$ into the sets $\mathfrak{M}_{x 1}^{1}$ and $\mathfrak{M}_{x 1}^{2}$. We determine the most general form of the scalar concomitant in $\mathfrak{M}_{x 1}^{1}$. Next we divide the set $\mathfrak{M}_{x 1}^{2}$ into two allowable subsets in which we determine the relative scalar concomitants of $t_{\lambda \mu}^{\nu}$.

With this aim we define the following objects:

$$
\mathfrak{a}^{\lambda} \stackrel{\mathrm{df}}{=}-\varepsilon^{\lambda e}{\stackrel{1}{v_{e}}}
$$

where $\varepsilon^{\lambda e}$ are Ricci symbols.

The sign "-" in definition (4.1) is used for the simplification of the further calculations.

The object $\mathfrak{a}^{\lambda}$ is a non-vanishing contravariant vector density

$$
\mathfrak{a}^{\lambda^{\prime}}=J^{-1} A_{\lambda}^{\lambda^{\prime}} \mathfrak{a}^{\lambda}
$$

where

$$
J=\operatorname{det}\left[A_{\lambda}^{\lambda^{\prime}}\right] \neq 0 .
$$

We now define the object $\mathfrak{h}^{p}$ :

$$
\mathfrak{h}^{\nu} \stackrel{\mathrm{d} f}{=} t_{\lambda \mu}^{\nu} \mathfrak{a}^{\lambda} \mathfrak{a}^{\mu}
$$

which, as can easily be verified, is a contravariant vector density of weight 2 :

$$
\mathfrak{h}^{\nu^{\prime}}=J^{-2} A_{v}^{v^{\prime}} \mathfrak{h}^{\nu} \text {. }
$$

Now, analogically to (4.1), we define the object $\mathfrak{h}_{\lambda}$ :

$$
\mathfrak{h}_{\lambda} \stackrel{\mathrm{df}}{=}-\varepsilon_{\lambda \varrho} \mathfrak{h}^{\varrho} \text {. }
$$


The object $\mathfrak{h}_{\lambda}$ is a covariant vector density of weight 1 :

$$
\mathfrak{h}_{\lambda^{\prime}}=J^{-1} A_{\lambda^{\prime}}^{\lambda} \mathfrak{h}_{\lambda} \text {. }
$$

Finally we define three objects, $\mathfrak{g}, \mathfrak{f}$ and $\mathfrak{w}$ :

$$
\begin{array}{r}
\mathfrak{g} \stackrel{d \mathfrak{d}=}{=} \mathfrak{h}^{\alpha} v_{a}, \\
\mathfrak{f} \stackrel{\text { df }}{=} t_{\lambda \mu}^{\nu} \mathfrak{h}^{\lambda} \mathfrak{h}^{\mu} v_{\nu}, \\
\mathfrak{w} \stackrel{\text { df }}{=} t_{\lambda \mu}^{\nu} \mathfrak{h}^{\lambda} \mathfrak{h}^{\mu} \mathfrak{h}_{\nu} .
\end{array}
$$

It is easy to see that the objects $\mathfrak{g}$ and $\mathfrak{f}$ are $W$-densities and $\mathfrak{w}$ is a $\dot{G}$-density, their weights being two, four and five, respectively:

$$
\mathfrak{g}^{\prime}=J^{-2} \mathfrak{g}, \quad \mathfrak{f}^{\prime}=J^{-4} \mathfrak{f}, \quad \mathfrak{w}^{\prime}=J^{-5} \mathfrak{w} .
$$

Now we divide the allowable set $\mathfrak{M}_{x 1}$ into two allowable subsets $\mathfrak{M}_{x 1}^{1}$ and $\mathfrak{M}_{x 1}^{2}$ :

$$
\begin{aligned}
& \mathfrak{M}_{\varkappa 1}^{1}=\left\{t_{\lambda \mu}^{\nu}: t_{\lambda \mu}^{\nu} \in \mathfrak{M}_{x 1}, \mathfrak{g} \neq 0\right\}, \\
& \mathfrak{M}_{\varkappa 1}^{2}=\left\{t_{\lambda \mu}^{\nu}: t_{\lambda \mu}^{\nu} \in \mathfrak{M}_{x 1}, \mathfrak{g}=0\right\} .
\end{aligned}
$$

We shall determine the relative scalar concomitants of the tensor $t_{\lambda_{\mu}}^{\nu}$ in these sets.

We consider the case $t_{\lambda \mu}^{v} \in \mathfrak{M}_{x 1}^{1}$. Let us take the transformation of the coordinate system, the parameters of which are determined as follows:

$$
A=\left\|A_{\lambda}^{\lambda^{\prime}}\right\| \stackrel{\mathrm{d} f}{=} \frac{1}{\mathfrak{g}}\left\|\begin{array}{cc}
1 & 1 \\
v_{1} & v_{2} \\
\mathfrak{h}_{1} & \mathfrak{h}_{2}
\end{array}\right\| .
$$

The inverse matrix is of the form

$$
A^{-1}=\left\|A_{\lambda^{\prime}}^{2}\right\|=\left\|\begin{array}{ll}
\mathfrak{h}^{1} & \mathfrak{a}^{1} \\
\mathfrak{h}^{2} & \mathfrak{a}^{2}
\end{array}\right\| .
$$

After such a change of the coordinate system the components of vectors $v_{\lambda^{\prime}}$ and $v_{\lambda^{\prime}}$ take the form

$$
\begin{array}{ll}
1 & 1 \\
v_{1^{\prime}}
\end{array}=\mathfrak{g}, \quad \stackrel{2}{v_{2^{\prime}}=0 ;} \quad \stackrel{2}{v_{1^{\prime}}=x \mathfrak{g},} \quad \stackrel{2}{v_{2^{\prime}}=0 .}
$$

On the basis of (4.15) we infer as a corollary that after the transformation of the coordinate system determined by (4.13) four components of $t_{\lambda \mu}^{v}$, namely $t_{1^{\prime} 2^{\prime}}^{1^{\prime}}, t_{1^{\prime} 2^{\prime}}^{2^{\prime}}, t_{2^{\prime} 1^{\prime}}^{1^{\prime}}$ and $t_{2^{\prime} 1^{\prime}}^{2^{\prime}}$, are expressed by the components $t_{1^{\prime} 1^{\prime}}^{1^{\prime}}$ and $t_{2^{\prime} 2^{\prime}}^{2^{\prime}}$ 
So the functional equation of the required scalar concomitants in $\mathfrak{M}_{x 1}^{1}$ can be written in the form

$$
f\left(t_{\lambda \mu}^{\nu}\right)=\varphi\left(\varkappa, \mathfrak{g}, \frac{\mathfrak{f}}{\mathfrak{g}}, \frac{\mathfrak{w}}{\mathfrak{g}}\right),
$$

where

$$
\begin{aligned}
& t_{1^{\prime \mathfrak{I}^{\prime}}}^{1^{\prime}}=\frac{1}{\mathfrak{g}} t_{\lambda \mu}^{\nu} \mathfrak{h}^{\lambda} \mathfrak{h}^{\mu} v_{\nu}=\frac{\mathfrak{f}}{\mathfrak{g}}, \\
& t_{\mathfrak{1}^{\prime} \mathfrak{1}^{\prime}}^{2^{\prime}}=\frac{1}{\mathfrak{g}} t_{\mu \mu}^{\nu} \mathfrak{h}^{\lambda} \mathfrak{h}^{\mu} \mathfrak{h}_{v}=\frac{\mathfrak{m}}{\mathfrak{g}}, \\
& t_{2^{\prime} \mathbf{2}^{\prime}}^{1^{\prime}}=\frac{1}{\mathfrak{g}} t_{\lambda \mu}^{\nu} \mathfrak{a}^{\lambda} \mathfrak{a}^{\mu} v_{\nu}^{1}=1, \\
& t_{2^{\prime} 2^{\prime}}^{2^{\prime}}=\frac{1}{\mathfrak{g}} t_{\lambda \mu}^{\nu} \mathfrak{a}^{\lambda} \mathfrak{a}^{\mu} \mathfrak{h}_{v}=0 .
\end{aligned}
$$

Let us now examine two cases: $\mathfrak{w} \neq 0$ and $\mathfrak{w}=0$. When $\mathfrak{w} \neq 0$, let us take the transformation of the coordinate system of the form $\xi^{\lambda^{\prime \prime}}=\xi^{\lambda^{\prime \prime}}\left(\xi^{\lambda^{\prime}}\right)$ for which

$$
\operatorname{det}\left\|A_{\lambda^{\prime}}^{\lambda^{\prime \prime}}\right\|=\operatorname{sgn} \mathfrak{w}|\mathfrak{g}|^{1 / 2} .
$$

holds.

After such a change of the coordinate system the right-hand side of (4.16) is of the form

$$
\varphi\left(\varkappa, \operatorname{sgng}, \frac{\mathfrak{f} \operatorname{sgn} \mathfrak{g}}{\mathfrak{g}^{2}}, \frac{|\mathfrak{w}| \operatorname{sgn} \mathfrak{g}}{|\mathfrak{g}|^{5 / 2}}\right),
$$

and it represents the general scalar" concomitants.

In the case $\mathfrak{w}=0$ it is enough to take the transformation of the coordinate system for which

$$
\operatorname{det}\left\|A_{\lambda^{\prime}}^{\lambda^{\prime \prime}}\right\|=|\mathfrak{g}|^{1 / 2}
$$

holds.

After such a change of the coordinate system we have

$$
\varphi\left(\varkappa, \mathfrak{g}, \frac{\mathfrak{f}}{\mathfrak{g}}\right)=\varphi\left(\varkappa, \operatorname{sgng}, \frac{\mathfrak{f} \text { sgng }}{\mathfrak{g}^{2}}\right) .
$$

We state a posteriori that (4.21) is a particular case of formula (4.13). Thus we have proved the following

THEOREM 2. The most general scalar concomitant of $t_{\lambda \mu}^{\nu}$ in $\mathfrak{M}_{x 1}^{1}$ is an arbitrary function of the form

$$
f\left(t_{\lambda \mu}^{\nu}\right)=\varphi\left(\varkappa, \text { sgng },-\frac{\mathfrak{f} \operatorname{sgn} \mathfrak{g}}{\mathfrak{g}^{2}}, \frac{|\mathfrak{w}| \text { sgng }}{|\mathfrak{g}|^{5 / 2}}\right),
$$


where $x, g, f$ and $\mathfrak{w}$ are defined in (2.4), (4.7), (4.8) and (4.9).

In the case $t_{\lambda \mu}^{\nu} \in \mathfrak{M}_{x]}^{2}$ we have the following relation:

$$
\mathfrak{g}=t_{\lambda_{\mu}}^{\nu} \mathfrak{a}^{\lambda} \mathfrak{a}^{\mu} v_{v}=\mathbf{0}
$$

If we write

$$
a_{\lambda \mu} \stackrel{\mathrm{df}}{=} t_{\lambda_{\mu}}^{\nu} \stackrel{1}{v_{\nu}},
$$

then relation (4.22) becomes:

$$
a_{\lambda \mu} a^{\lambda} a^{\mu}=0 .
$$

After a set of elementary but long calculations [12] it can be shown that if $\mathfrak{a}^{\lambda}$ (see (4.1)) fulfils (4.24), then the tensor $a_{\lambda \mu}$ is a symmetric one:

$$
a_{\mu \lambda}=a_{\lambda \mu} \text {. }
$$

Now we divide the set $\mathfrak{M}_{\varkappa 1}^{2}$ into two allowable subsets, $\mathfrak{M}_{x 1}^{21}$ and $\mathfrak{M}_{x 1}^{22}$ :

$$
\begin{aligned}
& \mathfrak{M}_{\varkappa 1}^{21}=\left\{t_{\lambda \mu}^{\nu}: t_{\lambda \mu}^{v} \in \mathfrak{M}_{\varkappa 1}^{2}, \operatorname{det}\left[a_{\lambda \mu}\right] \neq 0\right\}, \\
& \mathfrak{M}_{\varkappa 1}^{22}=\left\{t_{\lambda \mu}^{v}: t_{\lambda_{\mu}}^{v} \in \mathfrak{M}_{\varkappa 1}^{2}, \operatorname{det}\left[a_{\lambda \mu}\right]=0\right\} .
\end{aligned}
$$

In $\mathfrak{M}_{x 1}^{21}$ we can construct the tensor $a^{\lambda \mu}$ inverse to the tensor $a_{\lambda \mu}$, i.e. satisfying the relations ([2], p. 107):

$$
a^{\alpha \varrho} a_{\varrho \beta}=\delta_{\beta}^{\alpha} \text {. }
$$

Let us consider the following tensor in $\mathfrak{M}_{x 1}^{21}$ :

$$
b_{\lambda \mu} \stackrel{\mathrm{df}}{=} 2 t_{\lambda[1}^{[1} t_{2]_{\mu}^{2}} \text {. }
$$

The tensor $b_{\lambda_{\mu l}}$ is also a symmetric one. Let us form the transvection of $b_{\lambda_{\mu}}$ and $a^{\lambda \mu}$ :

$$
C_{\lambda}^{\mu} \stackrel{\mathrm{df}}{=} b_{\lambda_{\varrho}} a^{\varrho \mu}
$$

As can easily be verified, the trace of tensor $c_{\lambda}^{\mu}$ vanishes on the whole $\mathfrak{M}_{x 1}^{21}$. Let us write

$$
\tau \stackrel{\text { df }}{=} \operatorname{det}\left[c_{\lambda}^{\mu}\right] \text {. }
$$

TIIEOREM 3. The most general scalar concomitant of $t_{\lambda \mu}^{\nu}$ in $\mathfrak{M}_{x 1}^{21}$ is an arbitrary function of the form

$$
f\left(t_{\lambda \mu}^{v}\right)=\varphi(\varkappa, \tau),
$$

where $\tau$ is defined by (4.13) and $x$ by (2.4).

Proof. Since $\mathfrak{M}_{\varkappa 1}^{21} \subset \mathfrak{M}_{\varkappa 1}^{2} \subset \mathfrak{M}_{x 1}$, the relations

$$
\begin{gathered}
\stackrel{2}{2}_{v_{\lambda}}=\varkappa v_{\lambda}, \quad a_{\lambda \mu} \mathfrak{a}^{\lambda} \mathfrak{a}^{\mu}=0, \\
\operatorname{det}\left[a_{\lambda \mu}\right] \neq 0, \quad a_{\mu \lambda}=a_{\lambda \mu} .
\end{gathered}
$$

hold. 
Let us consider a coordinate system in which the components of the non-vanishing covariant vector $v_{\lambda}$ have the following values:

$$
\stackrel{1}{v_{1}} \stackrel{*}{=} 1, \quad \stackrel{1}{v_{2}} \stackrel{*}{=} 0
$$

(the $\operatorname{sign} \stackrel{*}{=}$ means that the equality does not hold in each allowable coordinate system). Expressing the components of vectors $v_{\lambda}$ and $v_{\lambda}$, the vector density $\mathfrak{a}^{\lambda}$ and the components of tensor $a_{\lambda \mu}$ occurring in (4.32) by the components of tensor $t_{\lambda \mu}^{\nu}$, we can write relations (4.32) using the coordinate system determined by (4.33) in the form

$$
\begin{gathered}
t_{12}^{2} \stackrel{*}{=} 1-t_{11}^{1}, \quad t_{21}^{1} \stackrel{*}{=} t_{12}^{1}, \quad t_{12}^{1} \stackrel{*}{\neq} 0, \\
t_{21}^{2} \stackrel{*}{=} x-t_{11}^{1}, \quad t_{22}^{1} \stackrel{*}{=} 0, \quad t_{22}^{2} \stackrel{*}{=}-t_{19}^{1} .
\end{gathered}
$$

Let us now take into consideration a transformation of the coordinate system which preserves relations (4.33) and (4.37) [13]. Let us denote the parameters of such a transformation by

$$
\left\|A_{\lambda}^{\lambda^{\prime}}\right\|=\left\|\begin{array}{ll}
1 & 0 \\
\gamma & \delta
\end{array}\right\|, \quad \delta \neq \mathbf{0} .
$$

Substituting in (4.35) the values of $\gamma$ and $\delta$,

$$
\gamma=\frac{1}{2} t_{11}^{1}, \quad \delta=t_{12}^{1},
$$

we get

$$
\begin{aligned}
& t_{1^{\prime} 1^{\prime}}^{1^{\prime}}=0, \quad t_{1^{\prime} 1^{\prime}}^{2^{\prime}}=-\tau, \quad t_{1^{\prime} 2^{\prime}}^{1^{\prime}}=1, \quad t_{1^{\prime} 2^{\prime}}^{2^{\prime}}=1, \\
& t_{2^{\prime} 1^{\prime}}^{1^{\prime}}=1, \quad t_{2^{\prime} 1^{\prime}}^{2^{\prime}}=\varkappa, \quad t_{2^{\prime} 2^{\prime}}^{1^{\prime}}=0, \quad t_{2^{\prime} 2^{\prime}}^{2^{\prime}}=-1 \text {. }
\end{aligned}
$$

Thus our theorem has been proved.

In the set $\mathfrak{M}_{x 1}^{22}$ (i.e. in the case where $\operatorname{det}\left[a_{\lambda \mu}\right]=0$ ) we can show [12] that the tensor $a_{\lambda \mu}$ is proportional to the product $v_{\lambda} v_{\mu}$, where $v_{\lambda}$ is the non-vanishing vector determined by (2.2),

$$
a_{\lambda \mu}=\stackrel{1}{\eta} v_{\dot{\lambda}} \cdot v_{\mu}
$$

where $\eta$ is the scalar.

Analogically to the proof of Theorem 3 we can prove [12] the following

THEOREM 4. The most general scalar concomitant of $t_{\lambda \mu}^{\nu}$ in $\mathfrak{M}_{\kappa 1}^{22}$ is an arbitrary function of the form

$$
f\left(t_{\lambda \mu}^{p}\right)=\varphi(\varkappa, \eta)
$$

where $x$ is defined by (2.4) and $\eta$ is determined by (4.38). 
5. The scalar concomitants of $t_{\lambda \mu}^{v}$ in $\mathfrak{M}_{10}$. Besides the vectors $v_{\lambda}=0$ and $v_{\lambda} \neq 0$ determined in (2.2), we form from the tensor $t_{\lambda \mu}^{p}$ the following auxiliary concomitants:

$$
\begin{aligned}
& \stackrel{2}{a}^{\lambda} \stackrel{d f}{=}-\varepsilon^{\lambda e} \stackrel{2}{v_{Q}}, \\
& \mathfrak{h}^{2} \stackrel{\mathrm{df}}{=} t_{\lambda \mu}^{\nu}{\stackrel{2}{\mathfrak{a}^{\lambda}} \mathfrak{a}^{\mu},}^{\mu}, \\
& \mathfrak{h}_{\lambda} \stackrel{\mathrm{df}}{=}-\varepsilon_{\lambda_{\ell}} \mathfrak{h}^{\mathbf{2}}, \\
& \mathfrak{g} \stackrel{\mathrm{df}}{=} \mathfrak{h}^{\alpha} v_{a}^{2}, \\
& \stackrel{2}{\mathfrak{f}} \stackrel{\mathrm{df}}{=} t_{\lambda \mu}^{\nu} \stackrel{2}{\mathfrak{h}}^{\lambda} \stackrel{2}{\mathfrak{h}}^{\mu}{ }^{2} v_{\nu} \text {, } \\
& \stackrel{2}{\mathfrak{m}} \stackrel{\mathrm{dP}}{=} t_{\lambda \mu}^{\nu} \mathfrak{h}^{\lambda} \mathfrak{h}^{\mu} \mathfrak{h}_{v}
\end{aligned}
$$

The transformation formulas of objects (5.1)-(5.6) are the same as the transformation formulas of the suitable objects determined in Section 4 in (4.1), (4.3), (4.5), (4.7), (4.8) and (4.9) and denoted there by the same letters without the index 2 above.

The index 2 above denotes that instead of the vector $v_{\lambda}$ we take the vector ${ }^{2} v_{\lambda}$.

Now we divide the allowable set $\mathfrak{M}_{10}$ into two allowable subsets $\mathfrak{M}_{10}^{1}$ and $\mathfrak{M}_{10}^{2}$,

$$
\begin{aligned}
& \mathfrak{M}_{10}^{1}=\left\{t_{\lambda \mu}^{\nu}: t_{\lambda \mu}^{\nu} \in \mathfrak{M}_{10}, \stackrel{2}{\mathfrak{g}} \neq 0\right\} \\
& \mathfrak{M}_{10}^{2}=\left\{t_{\lambda \mu}^{\nu}: t_{\lambda \mu}^{\nu} \in \mathfrak{M}_{10}, \stackrel{2}{\mathfrak{g}}=0\right\} .
\end{aligned}
$$

Then we can prove the following

THEOREM 5. The most general scalar concomitant of $t_{\lambda \mu}^{\nu}$ in $\mathfrak{M}_{10}^{1}$ is an arbitrary function of the form

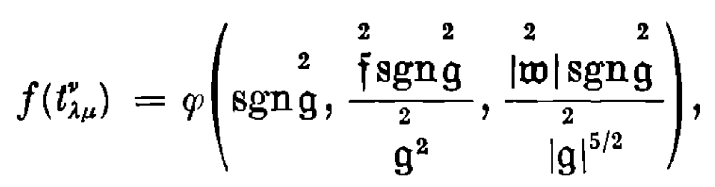

where $\stackrel{2}{g}, \stackrel{2}{f}$ and $\stackrel{2}{\mathfrak{w}}$ are defined by (5.4), (5.5) and (5.6).

The proof of this theorem is analogous to that of Theorem 2.

In the allowable set $\mathfrak{M}_{10}^{2}$ the following relation holds:

$$
\stackrel{2}{g}=t_{\lambda \mu}^{v} \mathfrak{a}^{2} \mathfrak{a}^{\mu} v_{v}^{2}=0 .
$$


Let us write

$$
\stackrel{2}{a_{\lambda \mu}} \stackrel{\mathrm{df}}{=} t_{\lambda \mu}^{\nu} \stackrel{2}{v_{\nu}} .
$$

It is easy to show that from condition (5.9) follows the symmetric

$$
\stackrel{2}{a_{\mu \lambda}}=\stackrel{2}{a_{\lambda \mu}} .
$$

Now we divide the allowable set $\mathfrak{M}_{10}^{2}$ into two further allowable subsets $\mathfrak{M}_{10}^{21}$ and $\mathfrak{M}_{10}^{22}$,

$$
\begin{aligned}
& \mathfrak{M}_{10}^{21}=\left\{t_{\lambda \mu}^{v}: t_{\lambda \mu}^{\nu} \in \mathfrak{M}_{10}^{2}, \operatorname{det}\left[a_{\lambda \mu}\right] \neq=0\right\} \\
& \mathfrak{M}_{10}^{22}=\left\{t_{\lambda \mu}^{v}: t_{\lambda \mu}^{\nu} \in \mathfrak{M}_{10}^{2}, \operatorname{det}\left[a_{\lambda \mu}\right]=0\right\} .
\end{aligned}
$$

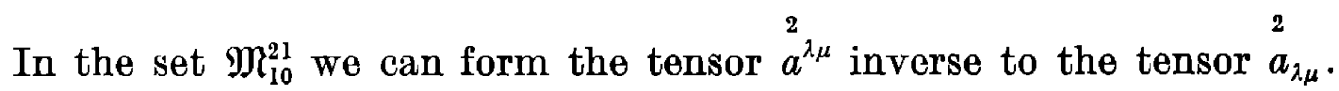
Still, let us consider in this set the tensor $b_{\lambda \mu}$ defined by (4.29). Let us form the transvection of $b_{\lambda \mu}$ and ${ }^{2} a^{\lambda \mu}$.

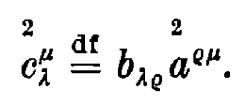

Let us put, further,

$$
\stackrel{2}{\tau} \stackrel{\mathrm{df}}{=} \operatorname{det}\left[c_{\lambda}^{\mu}\right]
$$

THEOREM 6. The most general scalar concomitant of $t_{\lambda \mu}^{\nu}$ in $\mathfrak{M}_{10}^{21}$ is an arbitrary function of the form

$$
f\left(t_{\lambda \mu}^{v}\right)=\varphi(\stackrel{2}{\tau}),
$$

where $\tau$ is defined by (5.15).

We omit the proof, which is analogous to that of Theorem 3 .

In the allowable set $\mathfrak{M}_{10}^{22}$ the relation

$$
\begin{aligned}
& 2 \\
& a_{\lambda_{\mu}}
\end{aligned}=\eta_{2} v_{\lambda} v_{\mu}
$$

holds, where ${ }^{2} v_{\lambda}$ is the vector defined in $(2.2), a_{\lambda \mu}$ the tensor defined in (5.11) and $\begin{aligned} & 2 \\ & \eta\end{aligned}$

THEOREM 7. The most general scalar concomitant of $t_{\lambda \mu}^{\nu}$ in $\mathfrak{M}_{10}^{22}$ is an arbitrary function of the form

$$
f\left(t_{\lambda \mu}^{p}\right)=\stackrel{2}{\varphi(\eta)}
$$

where ${ }^{2}$ is determined by (5.16).

8 - Annales Polonici Mathematici XXVH.1 
6. The scalar concomitants of $t_{\lambda \mu}^{\nu}$ in $\mathfrak{M}_{00}$. We shall reduce the determination of the scalar concomitants of $t_{\lambda \mu}^{v}$ in the set $\mathfrak{M}_{00}$ to the determination of the so-called transitive domains [3]. It will be shown that the allowable set $\mathfrak{M}_{00}$ falls into five transitive domains. On the basis of (2.2) and (2.6) the tensor $t_{\lambda \mu}^{\nu}$ belonging to the allowable set $\mathfrak{M}_{00}$ has only four essential components, $t_{11}^{1}, t_{11}^{2}, t_{22}^{1}$ and $t_{22}^{2}$; the remaining components can be expressed by $t_{11}$ and $t_{22}^{2}$. We have namely:

$$
t_{12}^{1}=-t_{22}^{2}, \quad t_{12}^{2}=-t_{11}^{1}, \quad t_{21}^{1}=-t_{22}^{2}, \quad t_{21}^{2}=-t_{11}^{1} .
$$

For brevity we adopt the following notation:

$$
z_{1}=t_{11}^{1}, \quad z_{2}=t_{11}^{2}, \quad z_{3}=t_{22}^{1}, \quad z_{4}=t_{22}^{2} .
$$

Let us consider the tensor $b_{\lambda \mu}$ defined by (4.29):

$$
b_{\lambda \mu}=2 t_{\lambda[1}^{[1} t_{2] \mu}^{2]} \text {. }
$$

Now we will express the components of $b_{\lambda_{\mu}}$ by the essential components of tensor $t_{\lambda \mu}^{\nu}$ taken from the allowable set $\mathfrak{M}_{00}$,

$$
\begin{aligned}
& b_{11}=z_{2} z_{4}-z_{1}^{2}, \\
& b_{12}=b_{21}=\frac{1}{2}\left(z_{1} z_{4}-z_{2} z_{3}\right), \\
& b_{22}=z_{1} z_{3}-z_{4}^{2} .
\end{aligned}
$$

The transitive domains of the symmetric tensor $b_{\lambda_{\mu}}$ determine the allowable subsets of $\mathfrak{M}_{00}$.

The transivity domains for an arbitrary twice covariant tensor in the two-dimensional space have been determined by $E$. Siwek in the paper [11]. Denoting the coordinates of the symmetric twice covariant tensor by $x_{i k}(i, k=1,2)$, we have the following transitive domains for it:

$$
\begin{aligned}
& \mathfrak{N}_{1}: x_{11}=x_{12}=x_{22}=0, \\
& \mathfrak{N}_{2}: x_{11} \geqslant 0, \quad x_{22} \geqslant 0, \quad x_{11}^{2}+x_{22}^{2}>0, \quad x_{11} x_{22}-x_{12}^{2}=0, \\
& \mathfrak{N}_{3}: x_{11} \leqslant 0, \quad x_{22} \leqslant 0, \quad x_{11}^{2}+x_{22}^{2}>0, \quad x_{11} x_{22}-x_{12}^{2}=0, \\
& \mathfrak{N}_{4}: x_{11}>0, \quad x_{22}>0, \quad x_{11} x_{22}-x_{12}^{2}>0, \\
& \mathfrak{N}_{5}: x_{11}<0, \quad x_{22}<0, \quad x_{11} x_{22}-x_{12}^{2}>0, \\
& \mathfrak{N}_{6}: x_{11} x_{22}-x_{12}^{2}<0 .
\end{aligned}
$$

If can be proved that the symmetric tensor $b_{\lambda^{\prime}}$ defined by (6.3) for the tensor $t_{\lambda \mu}^{p}$ taken from the allowable set $\mathfrak{M}_{00}$ cannot belong to the transitive domains $\mathfrak{N}_{2}$ and $\mathfrak{N}_{4}$.

If only $b_{\lambda \mu} \in \mathfrak{N}_{2}$, then, according to (6.5), the components of the tensor $b_{\lambda \mu}$ ought to satisfy the following relations:

$$
b_{11} \geqslant 0, \quad b_{22} \geqslant 0, \quad b_{11}^{2}+b_{22}^{2}>0, \quad b_{11} b_{22}-b_{12}^{2}=0 .
$$


It follows from (6.6) that the tensor $b_{\lambda \mu}$ can be put in the canonical form. Let

$$
b_{11} \stackrel{*}{=} 1, \quad b_{12} \stackrel{*}{=} 0, \quad b_{21} \stackrel{*}{=} 0, \quad b_{22} \stackrel{*}{=} 0 .
$$

Then on the basis of (6.4) we get the following system of equations:

$$
z_{2} z_{4}-z_{1}^{2} \stackrel{*}{=} 1, \quad z_{1} z_{4}-z_{2} z_{3} \stackrel{*}{=} 0, \quad z_{1} z_{3}-z_{4}^{2} \stackrel{*}{=} 0
$$

It can easily be shown that this system is contradictory. Analogically we can show that the tensor $b_{\lambda \mu}$ cannot fulfil the conditions which determine the transitive domains $\mathfrak{N}_{4}$.

In the case where $b_{\lambda_{\mu}} \in \mathfrak{N}_{1}$ we get, according to (6.3) and (6.4), the following system of equations:

$$
z_{2} z_{4}-z_{1}^{2}=0, \quad z_{1} z_{4}-z_{2} z_{3}=0, \quad z_{1} z_{3}-z_{4}^{2}=0 .
$$

If $z_{2}^{2}+z_{3}^{2}=0$, then the only solution of system $(6.9)$ is the vanishing one:

$$
z_{1}=z_{2}=z_{3}=z_{4}=0 \text {. }
$$

Hence it follows that all components of the tensor $t_{\lambda \mu}^{\nu}$ vanish. Of course it represents a transitive domain for the tensor $t_{\lambda_{\mu}}^{\nu}$ taken from the allowable set $\mathfrak{M}_{00}$. If

In the case where $z_{2}^{2}+z_{3}^{2}>0$ we get another solution of system (6.9).

$$
z_{1} \cdot z_{2} \neq 0
$$

then on the basis of the second equation of (6.9) we have

$$
z_{2}=k z_{1}, \quad z_{4}=k z_{3},
$$

where $k$ is an arbitrary real number. On the basis of (6.12) and of the third equation of (6.9) we get

$$
z_{1}=k^{2} z_{3}, \quad z_{2}=k^{3} z_{3}, \quad z_{4}=k z_{3} .
$$

Let us take into consideration the transformation of the coordinate system determined by the matrix

$$
\left\|A_{\lambda}^{\lambda^{\prime}}\right\| \stackrel{\mathrm{df}}{=}\left\|\begin{array}{cc}
\frac{1}{z_{3}} & 0 \\
-k & 1
\end{array}\right\| .
$$

Then on the basis of (2.1) and of (6.1) we get

$$
z_{1}^{\prime}=0, \quad z_{2}^{\prime}=0, \quad z_{3}^{\prime}=1, \quad z_{4}^{\prime}=0 .
$$

The cases $z_{1}=0$ or $z_{3}=0$ do not give anything new. In such cases it is easy to show the effective transformation of the coordinate system, after which the essential components of $t_{\lambda \mu}^{\nu}$ taken from the allowable set $\mathfrak{M}_{00}$ have the values (6.15). 
Thus we have proved

THEOREM 8. For the tensor $t_{\lambda \mu}^{\nu}$ from $\mathfrak{M}_{00}$ for which the tensor $b_{\lambda_{\mu}}$ determined by (6.3) belongs to the transitive domain $\mathfrak{N}_{1}$ we have two transitive domains determined by the conditions

$$
\begin{gathered}
\mathfrak{M}_{00}^{1}: z_{1}=z_{2}=z_{3}=z_{4}=0, \\
\mathfrak{M}_{00}^{2}: \begin{cases}z_{2} z_{4}-z_{1}^{2}=0, & z_{1} z_{4}-z_{2} z_{3}=0, \\
z_{1} z_{3}-z_{4}^{2}=0, & z_{2}^{2}+z_{3}^{2}=0,\end{cases}
\end{gathered}
$$

where $z_{i}(i=1,2,3,4)$ are the essential components of the tensor $t_{\lambda \mu}^{\prime \prime}$ in $\mathfrak{M}_{00}$ defined by (6.2). An arbitrary constant function on the domains $\mathfrak{M}_{00}^{1}$ and $\mathfrak{M}_{00}^{2}$ is a sclar concomitant.

In the case where $b_{\lambda_{\mu}} \in \mathfrak{N}_{3}$ the components of tensor $b_{\lambda_{\mu}}$ must satisfy in consequence of (6.5) the following relations:

$$
b_{11} \leqslant 0, \quad b_{22} \leqslant 0, \quad b_{11}^{2}+b_{22}^{2}>0, \quad b_{11} b_{22}-b_{12}^{2}=0 .
$$

In this case we can put the tensor $b_{\lambda \mu}$ in the canonical form [11]. Let

$$
b_{11} \stackrel{*}{=}-1, \quad b_{12}=b_{21} \stackrel{*}{=} 0, \quad b_{22} \stackrel{*}{=} 0 .
$$

On the basis of (6.4) and of (6.17) we get the following system of equations:

$$
z_{2} z_{4}-z_{1}^{2} \stackrel{*}{=}-1, \quad z_{1} z_{4}-z_{2} z_{3} \stackrel{*}{=} 0, \quad z_{1} z_{3}-z_{4}^{2} \stackrel{*}{=} 0 .
$$

Solving this system we obtain the solutions

$$
z_{1} \stackrel{*}{=} \pm 1, \quad z_{2} \stackrel{*}{=} c, \quad z_{3} \stackrel{*}{=} 0, \quad z_{4} \stackrel{*}{=} 0
$$

where $c$ is an arbitrary real number.

Let us take the transformation of the coordinate system defined by the matrix

$$
\left\|A_{\lambda^{\prime}}^{\lambda}\right\|=\left\|\begin{array}{ll}
\varepsilon & 0 \\
\gamma & \delta
\end{array}\right\|, \quad \varepsilon= \pm 1, \delta \neq 00 .
$$

This transformation preserves the canonical form (6.17) of the tensor $b_{\lambda \mu}$ [13]. Substituting in the matrix (6.20) the values

$$
\varepsilon=\operatorname{sgn} z_{1}, \quad \gamma=\frac{\varepsilon z_{2}}{3},
$$

we get in the new coordinate system the following values for the components of tensor $t_{\lambda \mu}^{v}$ taken from the allowable set $\mathfrak{M}_{00}$ :

$$
z_{1}^{\prime}=1, \quad z_{2}^{\prime}=0, \quad z_{3}^{\prime}=0, \quad z_{4}^{\prime}=0 .
$$

So we have got the third transitive domain $\mathfrak{M}_{00}^{3}$ for the tensor $t_{\lambda \mu}^{\nu}$ taken from the allowable set $\mathfrak{M}_{00}$. 
Thus we have proved the following

THEOREM 9. For the tensor tri from $\mathfrak{M}_{00}$ for which the tensor $b_{\lambda \mu}$, determined by (6.3), belongs to the transitive domain $\mathfrak{N}_{3}$ we have the transitive domain determined by the conditions

$$
\mathfrak{M}_{00}^{3}:\left\{\begin{array}{l}
z_{1} z_{4}-z_{1}^{2} \leqslant 0, \quad z_{1} z_{3}-z_{4}^{2} \leqslant 0, \\
\left(z_{2} z_{4}-z_{1}^{2}\right)^{2}+\left(z_{1} z_{3}-z_{4}^{2}\right)^{2}>0, \\
4\left(z_{2} z_{4}-z_{1}^{2}\right)\left(z_{1} z_{3}-z_{4}^{2}\right)=\left(z_{1} z_{4}-z_{2} z_{3}\right)^{2},
\end{array}\right.
$$

where $z_{i}(i=1,2,3,4)$ are the essential components of the tensor $t_{\lambda \mu}^{v}$ in $\mathfrak{M}_{00}$. An arbitrary constant function is a scalar concomitant in this domain.

Analogically to Theorem 9 we can prove the next two theorems, the proofs of which can be found in [12].

THEOREM 10. For the tensor $t_{\lambda \mu}^{\nu}$ from $\mathfrak{M}_{00}$ for which the tensor $b_{\lambda \mu}$, defined by (6.3), belongs to the transitive domain $\mathfrak{N}_{5}$ we have the transitive domain determined by the conditions

$$
\mathfrak{M}_{00}^{4}: \quad\left\{\begin{array}{l}
z_{2} z_{1}-z_{1}^{2}<0, \quad z_{1} z_{3}-z_{4}^{2}<0, \\
4\left(z_{2} z_{4}-z_{1}^{2}\right)\left(z_{1} z_{3}-z_{4}^{2}\right)>\left(z_{1} z_{4}-z_{2} z_{3}\right)^{2},
\end{array}\right.
$$

where $z_{i}(i=1,2,3,4)$ are the essential components of the tensor $t_{\lambda \mu}^{\nu} i_{i} \mathfrak{M}_{00}$. An arbitrary constant function is a scalar concomitant in this domain.

THEOREM 11. For the tensor $t_{\lambda \mu}^{\prime \prime}$ from $\mathfrak{M}_{00}$ for which the tensor $b_{\lambda \mu}$, defined by (6.3), belongs to the transitive domain $\mathfrak{N}_{6}$ we have the transitive domain determined by the conditions

$$
\mathfrak{M}_{00}^{5}: 4\left(z_{2} z_{4}-z_{1}^{2}\right)\left(z_{1} z_{3}-z_{4}^{2}\right)<\left(z_{1} z_{4}-z_{2} z_{3}\right)^{2},
$$

where $z_{i}(i=1,2,3,4)$ are the essential components of the tensor $t_{\lambda_{\mu}}$ in $\mathfrak{M}_{00}$. An arbitrary constant function is a scalar concomitant in this domain.

7. Remarks concerning the density concomitants of a tensor $t_{\lambda \mu}^{\nu}$ in a 2-dimensional space. On the basis of [1] it is known that the general density concomitant of object (1.1) is the product of a particular nonvanishing density concomitant and a general scalar concomitant of this object. So in those allowable sets of the tensor $t_{\lambda \mu}^{\nu}$ in which we have the non-vanishing density obtained from the tensor $t_{\lambda \mu \mu}^{\nu}$ we can determine the general density concomitant.

In the allowable set $\mathfrak{M}_{2}$ the determinant of the components of $v_{\lambda}$ 2

and $v_{\lambda}$ is the particular non-vanishing density concomitant,

$$
\mathfrak{v} \stackrel{\mathrm{d} \mathrm{f}}{=} \operatorname{det}\left\|\stackrel{e}{v_{\alpha}}\right\| \quad(a, \underline{e}=1,2) .
$$

It is known that $\mathfrak{v}$ is an ordinary density of weight 1 , and $|\mathfrak{w}|$ is a Weyl density of the same weight. 
Thus we have the following

THEOREM 12. The most general density concomitant of weight $(-p)$ of the tensor $t_{\lambda \mu}^{\nu}$ in the allowable set $\mathfrak{M}_{2}$ is of the form

wherc

$$
f\left(t_{\lambda \mu}^{v}\right)=\varepsilon|\mathfrak{v}|^{p} \varphi\left(\begin{array}{cccc}
1 & 2 & 1 & 2 \\
(\omega, & \omega, & \omega, & \omega \\
11 & & 22
\end{array}\right)
$$

$$
\varepsilon=\left\{\begin{array}{cl}
1 & \text { for } W \text {-density } \\
\operatorname{sgn} \mathfrak{v} & \text { for G-density }
\end{array}\right.
$$

and $\varphi$ is an arbitrary function of 4 variables.

In the allowable set $\mathfrak{M}_{x 1}^{1}$ the object $\mathfrak{g}$ defined by (4.7) is the particular non-vanishing $W$-density concomitant.

Thus we have the following

Theorem 13. The most general W-density concomitant of weight $(-p)$ of the tensor $t_{\lambda_{\mu}}^{\nu}$ in the allowable set $\mathfrak{M}_{x 1}^{1}$ is of the form

$$
f\left(t_{\lambda \mu}^{\nu}\right)=|\mathfrak{g}|^{p / 2} q\left(\varkappa, \operatorname{sgn} \mathfrak{g}, \frac{\mathfrak{f} \operatorname{sgn} \mathfrak{g}}{\mathfrak{g}^{2}}, \frac{|\mathfrak{w}| \operatorname{sgn} \mathfrak{g}}{|\mathfrak{g}|^{5 / 2}}\right),
$$

where $p$ is an arbitrary function.

In the allowable set $\mathfrak{M}_{x 1}^{21}$ the determinant of the components of the tensor $a_{\lambda \mu}$, defined by $(4.23)$, is the particular non-vanishin $W$-density concomitant.

THEOREM 14. The most general $W$-density concomitant of weight (-- $p$ ) of the tensor $t_{\lambda \mu}^{\nu}$ in the allowable set $\mathfrak{M}_{x 1}^{21}$ is of the form

$$
f\left(t_{\lambda \mu}^{\nu}\right)=\left|\operatorname{det}\left[a_{\lambda \mu}\right]\right|^{p / 2} \varphi(\varkappa, \tau),
$$

where $\varphi$ is an arbitrary function.

In the transitive domains $\mathfrak{M}_{00}^{4}$ and $\mathfrak{M}_{00}^{5}$ the determinant of the components of the tensor $b_{\lambda \mu}$, defined by (6.3), is the particular non-vanishing $W$-density concomitant.

Thus we have the following

Theorem 15. The most general $W$-density concomitant of weight $(-p)$ of the tensor $t_{\lambda_{\mu}}^{\nu}$ in the transitive domains $\mathfrak{M}_{00}^{4}$ and $\mathfrak{M}_{00}^{5}$ is of the form

$$
f\left(t_{\lambda \mu}^{p}\right)=C \cdot\left|\operatorname{det}\left[b_{\lambda \mu}\right]\right|^{p / 2},
$$

where $C$ is an arbitrary real number.

We shall deal with the problem of determination of all density concomitants of the tensor $t_{\lambda \mu}^{\nu}$ in the next paper. 


\section{References}

[1] J. Aczél and M. Hosszú, On conocomitants of mixed tensors, Ann. Polon. Math. 13 (1963), p. 163 - 171.

[2] S. Gołąb, Rachunek tensorowy, Warszawa 1966.

[3] - und M. Kucharzewski, Ein Beitrag zur Komitantentheorie, Acta Math. Acad. Hung. (1960), p. $173-174$.

[4] M. Kucharzewski, Ưber die skalaren Komitanten der Vektorfelder, Ann. Polon. Math. 9 (1961), p. 311 - 323.

[5] - Über die Vektorkomitanten der Vektorfelder, ibidem 9 (1961), p. 299 - 309.

[6] - Die skalaren Komitanten welche aus kovarianten und kontravarianten Vektoren gebildet sind, Tensor N.S. 12 (1962), p. 158 - 166.

[7] - Die kovarianten Vektorkomitanten die aus kontravarianten gebildet sind, ibidem 12 (1962), p. $140 \cdot 150$.

[8] - Zum Begriff der Komitante, Ann. Polon. Math. 13 (1963), p. $115-120$.

[9] - and M. Kuczma, Basic concept of the theory of geometric objects, Rozprawy Matematyczne 43, Warszawa 1964.

[10] - und A. Zajtz, Klassifikation der linearen homogenen geometrischen Objekte deren Komponentenzahl die Dimension des Raumes nicht übertrifft, Colloq. Math. 16 (1967), p. $185-192$.

[11] E. Siwek, Sur les domaines de transitivité du groupe de transformations des composants d'un tenseur covariant du second ordre, Ann. Polon. Math. 10 (1961), p. $217-224$.

[12] S. Wegrzynowski, Komitanty skalarne tensora o walencji $(1,2) w$ przestrzeni dwuwymiarowej, Zesz. Nauk. Polit. Szczec. Prace Mon. 61 (1970), p. 1 - 46.

[13] A. Zajtz, Algebraic Objects, Zesz Nauk. UJ. Prace Mat. 12 (1968), p. 67 - 79.

Reçu par la Rédaction le 31. 5. 1971 